\title{
ESTRUCTURA, COMPOSICIÓN Y DIVERSIDAD DEL MATORRAL DE DUNA COSTERA EN EL LITORAL YUCATECO
}

\author{
Wendy Torres, Martha Méndez, Alfredo Dorantes y Rafael Durán ${ }^{1}$ \\ Centro de Investigación Científica de Yucatán A.C. \\ 'Autor para la correspondencia: rduran@cicy.mx
}

\begin{abstract}
Resumen: Con el fin de brindar elementos para la toma de decisiones en futuros programas de manejo, se caracterizó la vegetación del matorral de duna costera a lo largo del litoral Yucateco, ya que es un ecosistema que ha sufrido un importante deterioro y fragmentación del hábitat debido a actividades antropogénicas. Se registró la altura, cobertura e identidad taxonómica de los individuos $\geq 50 \mathrm{~cm}$ de altura, en 10 localidades. En total se registraron 71 especies de plantas vasculares pertenecientes a 36 familias. Se detectaron comunidades de dos tipos, aquellas con una estructura arbustiva e individuos menores a 3 m; y aquellas con vegetación arbórea e individuos de mayor altura. Se determinaron los valores de diversidad alfa (26.6), gamma (71) y beta (2.66). Mediante el análisis de clasificación y ordenación se distinguieron tres grupos de vegetación: G1) en la parte oeste del litoral teniendo como especies importantes Pithecellobium keyense y Sideroxylon americanum, G2) en la parte centro Coccothrinax readii, Bravaisia berlandieriana y Metopium brownei, G3) en la parte este Coccothrinax readii, Thrinax radiata y Pseudophoenix sargenti. El estudio permitió documentar la heterogeneidad florística y estructural de las comunidades de dunas a lo largo del litoral y explorar algunas variables que contribuyan a explicarla.
\end{abstract}

Palabras claves: vegetación de duna costera, Yucatán, diversidad florística.

\begin{abstract}
In order to provide elements to make decisions in future management programs, the scrub vegetation of coastal sand dune was characterized in Yucatan, Mexico. This ecosystem has been deteriorated and fragmented due to anthropogenic activities. We recorded the height, coverage and taxonomic identity of individuals $\geq 50 \mathrm{~cm}$ in height, in ten locations. A total of 71 species of vascular plants in 36 families were recorded. Two types of communities, one with a shrub dominance and individuals lees than $3 \mathrm{~m}$ and other one with larger individuals $\geq 3 \mathrm{~m}$. Values of alpha diversity (26.6), gamma (71) and beta (2.66) were determined. Classification and ordenation analyzes distinguished three groups of vegetation: G1) in the western part of the coast was characterized by species such as Pithecellobium keyense and Sideroxylon americanum, G2) in the central part by Coccothrinax readii, Bravaisia berlandieriana and Metopium brownei, G3) in the east, was characterized by Coccothrinax readii, Thrinax radiata and Pseudophoenix sargentii. This study provides ecological elements to take decisions on future programs of restoration and management of the coastal dune in Yucatan, Mexico.
\end{abstract}

Key words: Coastal dune vegetation, Yucatan, floristic diversity.

$\mathbf{L}$ as comunidades vegetales de dunas costeras se encuentran distribuidas a lo largo de los litorales de todo nuestro país (Moreno-Casasola et al., 1998). En el estado de Yucatán, estas comunidades se distribuyen naturalmente en poco menos del $60 \%$ de su litoral y se estima que este ecosistema ha perdido aproximadamente la mitad de su territorio original (Durán et al., 2007). Actualmente se presenta como un mosaico de comunidades en diferentes estados de desarrollo o etapas serales, siendo éstas las condiciones en las que se encuentran la mayoría de las dunas costeras de México debido a su deterioro y fragmentación como producto de un crecimien- to urbano inmoderado, el establecimiento de casas de verano, hoteles, basureros clandestinos, carreteras y por la incidencia de huracanes, entre otros (Castillo et al., 1991).

En Yucatán la vegetación de dunas costeras tiene una composición florística interesante ya que comparte numerosos elementos con la flora de las Antillas Mayores y Menores, Florida, el sur de México y Centro América (Espejel, 1986). La península de Yucatán ocupa el segundo lugar con mayor número de especies endémicas de la flora costera de México, sólo superada por la península de Baja California (Moreno-Casasola et al., 1998). 
Actualmente se reconoce que estas comunidades aportan importantes servicios ambientales a la sociedad, siendo el más relevante su función como barrera de protección a la acción de vientos y mareas, protegiendo a las construcciones en caso de tormentas tropicales y huracanes (Miller et al., 2010). También contribuyen a la formación de suelo mediante la acumulación de arena por medio de sus raíces y la materia orgánica de su follaje, evitando la erosión (Wolfe y Nickling, 1993). Además, estas comunidades vegetales proveen refugio, alimento y protección a numerosas especies de insectos, reptiles, aves y mamíferos (Martínez et al., 1993; Flores y Espejel, 1994; Torres y Bojórquez, 2002; Durán et $a l$., en prensa).

Cabe señalar que a lo largo de las costas de México las comunidades de dunas presentan una alta diversidad florística y estructural como resultado de la heterogeneidad del paisaje, mismo que está influenciado por las diferencias hidrológicas, climáticas y edáficas que se manifiestan en el litoral. Miranda (1978) y Rzedowski (1981) consideran a las comunidades vegetales de dunas costeras como un tipo de vegetación halófito, debido a que se desarrollan en suelos con alto contenido de sales solubles. Espejel (1984) clasificó la vegetación de dunas de Yucatán en dos tipos, en función de los rangos de tolerancia a los factores físicos y bióticos a los que se encuentran expuestos los individuos, considerando su cercanía al mar. El primer tipo es considerado pionero, ubicándose cerca de la línea de costa, donde la arena tiene una gran movilidad y se caracteriza por la presencia de especies herbáceas y arbustivas de pequeña talla, más tolerantes a las condiciones ambientales extremas. El segundo tipo de duna es considerado un matorral, encontrándose hacia el interior, donde la arena está fija al sustrato y la vegetación alcanza una mayor altura.

Moreno-Casasola et al. (1998), así como Lortie y Cushman (2007) han apuntado como factores físicos y bióticos responsables de la configuración del paisaje de los sistemas de dunas, al viento, el agua y a la interacción con diferentes microorganismos. Señalan, que estos factores varían de acuerdo a la cercanía del mar, así como a las diferencias topográficas y edáficas que se encuentran en estos sistemas, generando la heterogeneidad de las comunidades vegetales que se desarrollan en las dunas.

Por su parte, Rzedowski (1981) menciona que el clima juega un papel preponderante en la heterogeneidad manifiesta en la composición y estructura de las comunidades vegetales, debido a que ejerce una influencia directa sobre las plantas y de forma indirecta en la formación del suelo, la conformación de la topografía y la distribución de la fauna y los microorganismos. Así, diferencias en clima, origen del sustrato y topografía local pueden tener implicaciones importantes en la determinación de la composición florística de cada sitio en particular, produciendo importantes diferencias de una región a otra (Moreno-Casasola et al., 1998).

Este estudio tiene por objetivo la caracterización florís- tica y estructural de la vegetación de dunas costeras a lo largo del litoral yucateco, así como conocer cuáles son los factores del medio que determinan su variación en composición y estructura. Esto permitirá brindar elementos para la toma de decisiones que apoyen la conservación y el manejo sustentable en esta zona del estado de Yucatán.

\section{Material y métodos}

Área de estudio. El estado de Yucatán cuenta con un litoral de aproximadamente $345 \mathrm{~km}$ de longitud (Euán-Ávila et al., en prensa), presentando ecosistemas de manglares y dunas costeras asociados a esteros y lagunas costeras (Duch-Gary, 1988, 1991; Flores et al., 1995). Las dunas costeras ocupan aproximadamente $205 \mathrm{~km}$ entre el Golfo de México y el Mar Caribe en localidades como Celestún, Progreso, Sisal, Telchac, Dzilam de Bravo, Río Lagartos y El Cuyo (Campos-Rios y Durán, 1991).

La zona costera se caracteriza por presentar una franja de clima del tipo BS, con algunas variantes que se caracterizan por tener escasas lluvias y altas temperaturas, presentando su parte más amplia en la zona de Progreso y los subtipos de climas registrados a lo largo del litoral son: BSo (h') (x') i, BSo (h') (e), BSo (h') w" i y BS1 (h') w" (i) (Orellana et al., 1999). De acuerdo con el Sistema Meteorológico Nacional, a partir de los registros de 1971-2000, la parte más seca de esta franja climática se encuentra en Progreso, con una precipitación de $687 \mathrm{~mm}$, siendo la estación de El Cuyo donde se registra la mayor precipitación, con $779 \mathrm{~mm}$ anuales. La temperatura promedio anual es de $25.8^{\circ} \mathrm{C}$ en Progreso y de $26.8^{\circ} \mathrm{C}$ en El Cuyo (SMN-CNA, 2007). La geología superficial está representada por los extensos depósitos conchíferos del cuaternario, principalmente del holoceno que forman las playas arenosas y las cuencas de sedimentación palustre en las ciénegas y esteros (Duch-Gary, 1991). Se caracteriza por la dominancia de suelos de color blanco-amarillento, arenosos y profundos, los cuales corresponden a regosoles calcáreos, según la FAO/UNESCO por su escaso desarrollo y por el alto contenido de calcio en la matriz mineral (DuchGary, 1988 y 1991).

Metodología. Se seleccionaron diez sitios en el matorral de duna costera que presentaron un estado conservado, para lo cual se utilizó como referencia el mapa de vegetación del ordenamiento territorial costero (Durán et al., 2007), donde se muestran los grados de perturbación de la vegetación de duna costera. Estos sitios fueron: Celestún, Sisal, Chuburná, Tulix, La Casona, San Benito, Holchit, Punta Cancunito, Alegrías y El Cuyo (Figura 1).

En cada sitio de muestreo se establecieron seis cuadrantes de $5 \times 5 \mathrm{~m}$, totalizando 60 unidades de muestreo. En ellos se registró la identidad taxonómica, la altura y la cobertura de aquellos individuos mayores de $50 \mathrm{~cm}$ de altura. Los ejemplares de respaldo colectados fueron depositados 


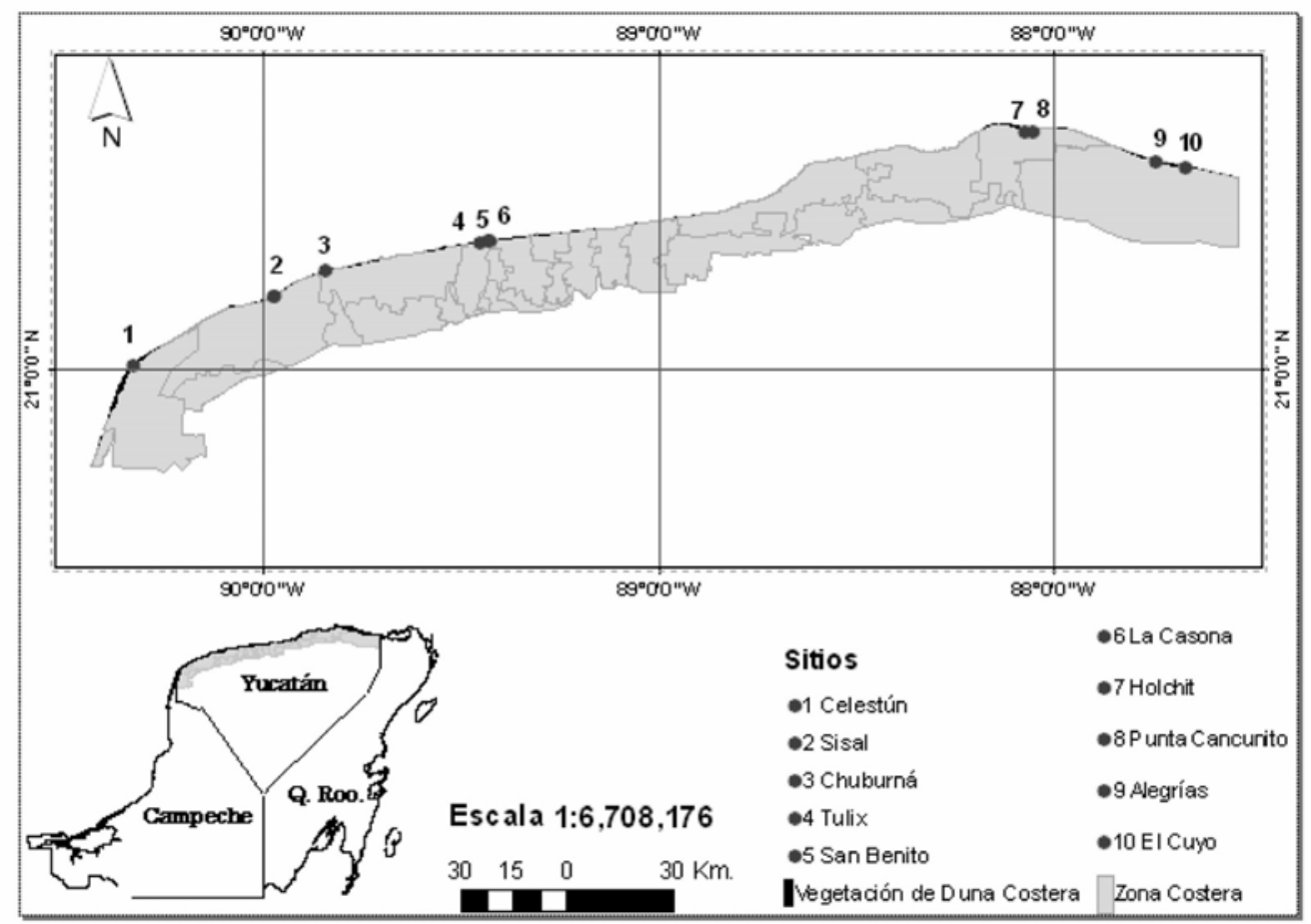

Figura 1. Ubicación de los sitios de muestreo

en el herbario CICY. La altura se midió utilizando un flexómetro para los individuos menores de un metro y una barra telescópica para los individuos de mayor talla. La cobertura se obtuvo midiendo dos diámetros perpendiculares de la copa de la planta, calculando su valor mediante la suma de los dos diámetros entre cuatro, al cuadrado por $\pi$ (MullerDombois y Ellenberg, 1974)

Mediante el método de Curtis (Curtis y McIntosh, 1951) se determinó el valor de importancia relativa de las especies (VIR). Asimismo, se analizó la diversidad de la comunidad con el índice de Shanon-Wiener (utilizando el logaritmo natural) y la equitatividad $\left(\mathrm{H} / \mathrm{H}_{\max }\right)$ (Magurran, 1988), y a partir de éstos se calculó el número de especies aparentes, de acuerdo con la propuesta de Hill (1973), modificado por Durán (1995). También se analizó la diversidad alfa, beta y gamma de las comunidades. La diversidad alfa se determinó mediante el promedio del número total de especies por localidad, la diversidad gamma se obtuvo mediante el registro del número de especies presentes en todas las localidades y la diversidad beta se calculó al dividir la gamma entre la diversidad alfa (Whittaker, 1972; Halffter, 1998; Primack et al., 2001)

La similitud entre las comunidades se estimó a través del índice de Sørensen (Muller-Dombois y Ellenberg, 1974) utilizando los valores de importancia relativa de las especies presentes en cada una de las localidades.

Se realizó un análisis de clasificación de las comunidades, mediante el análisis de agrupamientos, utilizando el
Programa PC-ORD4 (MacCune y Mefford, 1999). Para ello, la matriz de datos se generó tomando en consideración el VIR de las especies de cada cuadrante. Se eligió el índice de Sørensen (1948) como medida de distancia para definir la similitud, siendo éste el más robusto para datos ecológicos; a este análisis se le aplicó el método de Procesamientos de Permutaciones para Respuestas Múltiples (MRPP) para evaluar el grado de significancia de los grupos. También se realizó una ordenación indirecta mediante el análisis de escalamiento multidimensional no métrico (NMDS). En este análisis se utilizó la misma matriz de datos que se empleó en la clasificación y se construyó otra matriz con los promedios anuales de algunos elementos del clima (temperatura máxima, media y mínima, precipitación y oscilación térmica), obtenidos de la base de datos del Programa de Ordenamiento Ecológico del Territorio Costero de Yucatán (Euán-Ávila et al., 2007). Con el fin de determinar si la ordenación de los diferentes grupos de vegetación está relacionada con estos elementos del clima, se llevó a cabo la correlación entre los valores de los ejes obtenidos de cada cuadro y la matriz de valores de los elementos del clima registrados para cada cuadro de muestreo. Este análisis se realizó mediante el programa PC-ORD4 (MacCune y Mefford, 1999).

\section{Resultados}

Composición florística. En total se registraron 71 especies, correspondientes a 66 géneros y 36 familias, de las cuales 


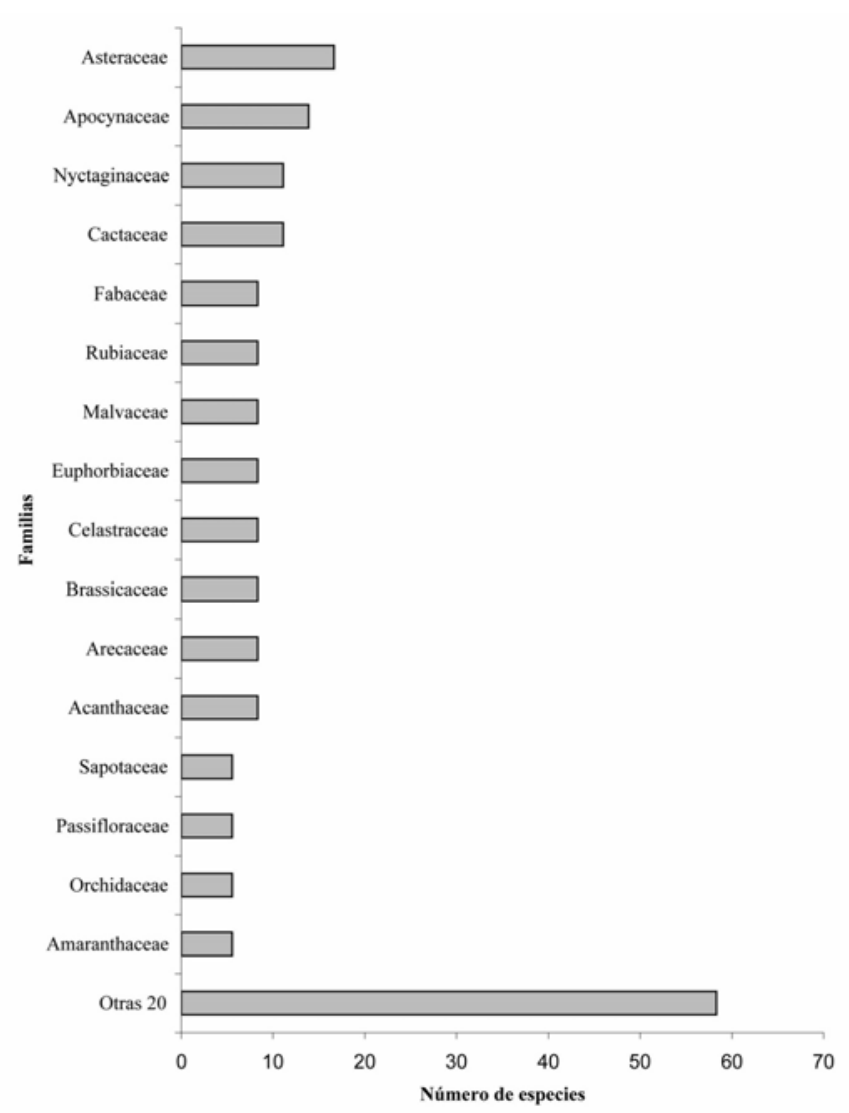

Figura 2. Distribución de las especies de las familias más diversas en diez localidades del matorral de duna costera de Yucatán.

29 especies corresponden a especies arbustivas, 13 árboles, tres especies de palmas, 17 herbáceas, una graminoide, seis lianas y dos epífitas. La familia Asteraceae es la mejor representada con un total de seis especies, seguida por la familia Nyctaginaceae representada por cuatro especies (Figura 2).
De las especies registradas, cuatro son endémicas de la Península de Yucatán: Coccothrinax readii, Nopalea gaumeri, Selenicereus donkelaarii y Diospyros cuneata. Sin embargo, también se encontraron dos especies endémicas que no fueron incorporadas en el análisis; Mammillaria gaumeri que no alcanza la talla mínima y Pilosocereus gaumeri observada fuera de los cuadros de muestreo.

Estructura. El cuadro 1 muestra la densidad de individuos registrados, la cobertura y la altura promedio para cada una de las comunidades analizadas. La localidad que registró la mayor densidad de individuos fue Tulix (8166.67 ind/ha), seguida por la localidad de San Benito (7316.67 ind/ha), siendo la localidad de Sisal la que presentó menor densidad de individuos (2583.33 ind/ha).

El porcentaje de cobertura varió desde $85.47 \%$ en la localidad El Cuyo, hasta $41.06 \%$ en la localidad de Chuburná, con un promedio del $61.60 \%$ y un coeficiente de variación de 27.35.

La altura de la vegetación varió a lo largo del litoral yucateco, presentándose comunidades tanto arbustivas como arbóreas, con alturas máximas que varían de $1.88 \mathrm{~m}$ a $6.53 \mathrm{~m}$, con alturas promedio que oscilan entre 0.94 y 1.92 (Cuadro 1). Las frecuencias de altura de los individuos nos permiten identificar dos patrones; uno donde el matorral es bajo, el cual se encuentra en la parte centro del litoral yucateco y donde los individuos únicamente alcanzan alturas de hasta $3 \mathrm{~m}$ y el otro presenta individuos de mayor talla, localizándose en los extremos este y oeste del litoral yucateco, con alturas de hasta $6.5 \mathrm{~m}$ (Figura 3). Algunas especies que se distribuyen ampliamente a lo largo de la costa varían en la talla que alcanzan en las diferentes localidades, tal es el caso de Coccothrinax readii cuya altura máxima varió de $2.25 \mathrm{~m}$ en San Benito (ubicado en el Centro del litoral) a $4.44 \mathrm{~m}$ en Alegrías (en el oriente del litoral). Las especies que

Cuadro 1. Estructura de la vegetación. Los subrayados se refieren a los valores mínimos y las negritas a los máximos. D.E= desviación estándar, C.V.= coeficiente de variación.

\begin{tabular}{|c|c|c|c|c|}
\hline Localidad & $\begin{array}{l}\text { Densidad } \\
\text { (No. Ind/ha) }\end{array}$ & $\begin{array}{c}\text { Cobertura } \\
(\%)\end{array}$ & $\begin{array}{l}\text { Altura máxima } \\
\qquad(\mathrm{m})\end{array}$ & $\begin{array}{l}\text { Altura promedio } \\
(\mathbf{m})\end{array}$ \\
\hline Celestún & 4800.00 & 83.61 & 4.60 & 1.59 \\
\hline Sisal & $\underline{2583.33}$ & 62.97 & 3.35 & 1.54 \\
\hline Chuburná & 3233.33 & $\underline{41.06}$ & $\underline{1.88}$ & $\underline{0.94}$ \\
\hline Tulix & 8166.67 & 46.06 & 3.00 & 1.36 \\
\hline San Benito & 7316.67 & 44.68 & 2.70 & 1.31 \\
\hline La Casona & 6550.00 & 44.76 & 3.00 & 1.11 \\
\hline Holchit & 4833.33 & 69.77 & 4.25 & 1.65 \\
\hline Punta Cancunito & 4583.33 & 61.88 & 4.95 & 1.65 \\
\hline Alegrías & 5400.00 & 75.71 & 6.53 & 1.75 \\
\hline El Cuyo & 5583.33 & 85.47 & 4.60 & 1.92 \\
\hline Promedio & 318.3 & 369.58 & 3.89 & 1.49 \\
\hline D.E & 103.09 & 101.09 & 1.36 & 0.30 \\
\hline C.V \% & 32.38 & 27.35 & 0.35 & 20.11 \\
\hline
\end{tabular}



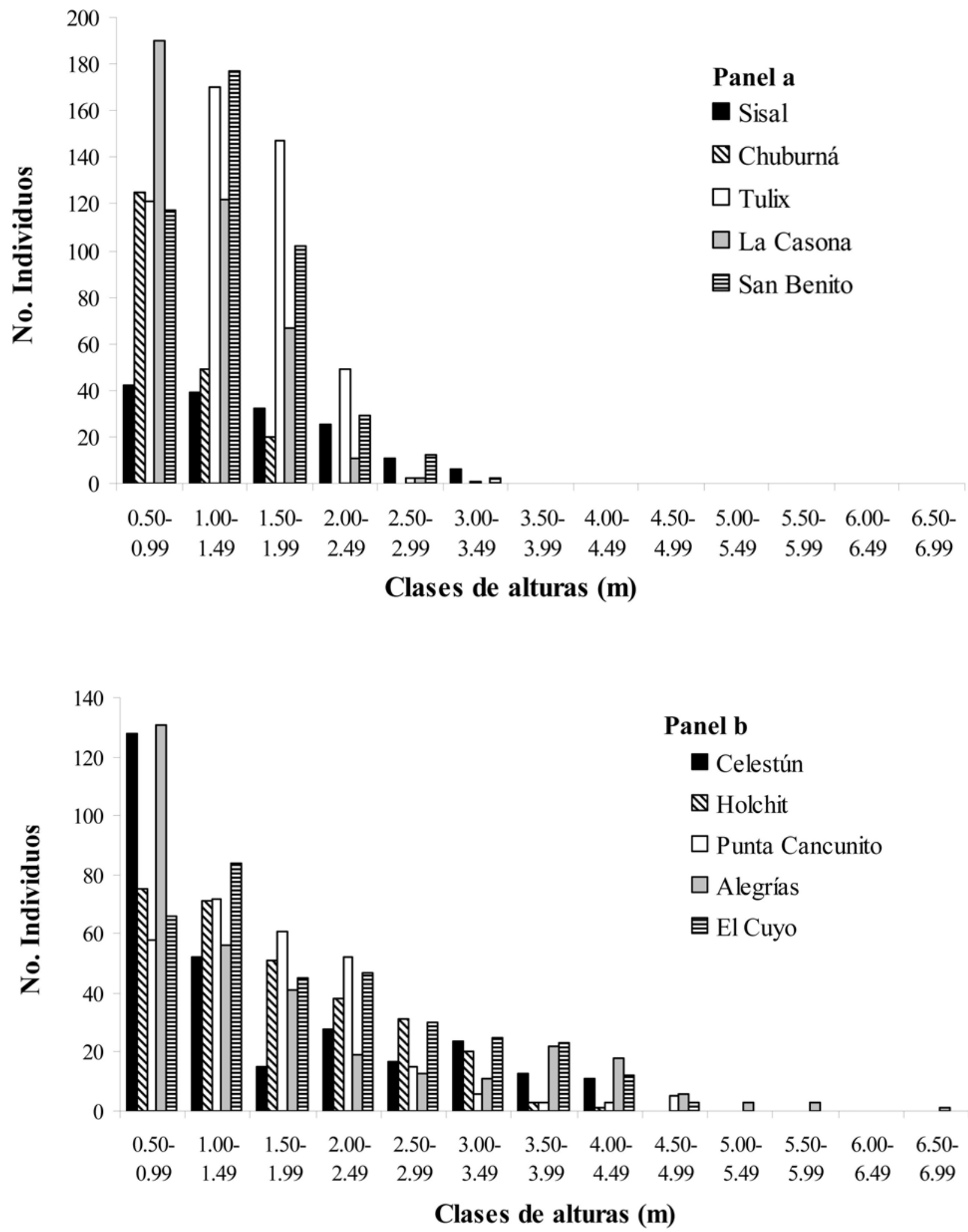

Figura 3. Patrones de altura de las comunidades muestreadas en la vegetación de duna costera del estado de Yucatán. Panel a comunidades de menor altura y panel $\mathbf{b}$ comunidades con mayor altura 
Cuadro 2. Valores de riqueza (S), número de Hill (N1), Shannon-Wiener (H), diversidad máxima (Hmax) y equitativilidad (E). Los valores en negritas son máximos y los subrayados mínimos. D.E= desviación estándar, C.V.= coeficiente de variación.

\begin{tabular}{|c|c|c|c|c|c|}
\hline Localidad & S & N1 & $\mathbf{H}$ & Hmax & E \\
\hline Celestún & 22 & 13.74 & 2.61 & 3.09 & 0.84 \\
\hline Sisal & 25 & 14.55 & 1.16 & 1.39 & 0.83 \\
\hline Chuburná & 26 & 16.18 & 2.78 & 3.25 & 0.85 \\
\hline Tulix & 29 & $\underline{8.95}$ & $\underline{0.95}$ & 1.46 & $\underline{0.65}$ \\
\hline San Benito & 33 & 12.50 & 1.09 & 1.51 & 0.72 \\
\hline La Casona & 31 & 16.29 & 1.21 & 1.49 & 0.81 \\
\hline Holchit & 22 & 9.22 & 0.96 & 1.34 & 0.71 \\
\hline Punta Cancunito & $\underline{19}$ & 9.33 & 0.97 & 1.27 & 0.75 \\
\hline Alegrías & 30 & 15.17 & 1.18 & 1.47 & 0.80 \\
\hline El Cuyo & 29 & 15.92 & 1.20 & 1.46 & 0.82 \\
\hline Promedio & 26.6 & 13.19 & 1.41 & 1.77 & 0.78 \\
\hline D.E & 4.55 & 3.00 & 0.68 & 0.74 & 0.07 \\
\hline C.V \% & 17.11 & 22.78 & 48.53 & 41.77 & 8.48 \\
\hline
\end{tabular}

presentaron mayor altura fueron Piscidia piscipula, Suriana maritima, Malpighia souzae, Manilkara zapota, Bursera simaruba, Pseudophoenix sargentii, entre otras.

Las diez especies que obtuvieron los mayores valores de importancia relativa (VIR) en cada una de las localidades se presentan en el apéndice 1: Coccothrinax readii fue la especie más importante en seis de las localidades, todas ellas ubicadas del centro al oriente del estado, con valores que oscilan entre 86.80 en Tulix a 40.83 en Alegrías. En las localidades del occidente las especies más importantes fueron Pithecellobium keyense, Bravaisia berlandieriana, Metopium brownei y Sideroxylon americanum. Otras especies importantes, sobretodo en la región oriente, fueron Thrinax radiata, Pseudophoenix sargentii, Enriquebeltrania crenatifolia y Gymnanthes lucida.

Diversidad. El promedio de especies por sitio fue de 26.6, en tanto que el número de especies aparentes promedio por localidad fue de 13.19. Punta Cancunito presentó el menor número de especies (19), en tanto que La Casona (31) y San Benito (33) presentaron los mayores valores. El valor promedio de diversidad de las comunidades, de acuerdo con el índice de Shannon, fue de 1.41, siendo las menos diversas las localidades Tulix y Holchit, en tanto que las más diversas fueron Chuburná y Celestún, esta última fue la localidad con el mayor valor de equitatividad (Cuadro 2). La diversidad alfa promedio obtenida para las 10 comunidades fue de 26.6 especies vegetales, en tanto que la diversidad gamma fue 71 especies, que representa la riqueza de especies presentes en el estudio, en tanto que la diversidad beta fue de 2.66.

Similitud. La localidades que presentaron mayor similitud fueron Holchit y Punta Cancunito con $96.10 \%$ de similitud, teniendo 18 especies en común, seguidas por las comunidades de San Benito y La Casona con una similitud del $94.06 \%$, compartiendo 26 especies. Las comunidades me-

Cuadro 3. Índice de similitud (Sørensen) de todas las comunidades muestreadas del matorral de duna costera de Yucatán. Los números en negritas son los de mayor similitud y los subrayados corresponden a los de menor similitud.

\begin{tabular}{|c|c|c|c|c|c|c|c|c|c|c|}
\hline Localidad & Celestún & Sisal & Chuburná & Tulix & San Benito & La Casona & Holchit & $\begin{array}{c}\text { Punta } \\
\text { Cancunito }\end{array}$ & Alegrías & El Cuyo \\
\hline Celestún & & 54.73 & 73.60 & 47.47 & 55.19 & 43.39 & 64.57 & 61.53 & 65.30 & 58.68 \\
\hline Sisal & & & 72.23 & 56.14 & 63.34 & 66.29 & 60.72 & 47.01 & 60.30 & 55.07 \\
\hline Chuburná & & & & 52.52 & 50.51 & 57.39 & 59.00 & 54.20 & 50.18 & $\underline{38.66}$ \\
\hline Tulix & & & & & 91.62 & 88.56 & 77.15 & 61.16 & 74.57 & 71.08 \\
\hline San Benito & & & & & & 94.06 & 71.76 & 59.75 & 73.99 & 66.82 \\
\hline La Casona & & & & & & & 66.85 & 58.54 & 61.22 & 58.37 \\
\hline Holchit & & & & & & & & 96.10 & 82.81 & 82.40 \\
\hline Punta Cancunito & & & & & & & & & 75.83 & 70.93 \\
\hline Alegrías & & & & & & & & & & 91.47 \\
\hline El Cuyo & & & & & & & & & & \\
\hline
\end{tabular}




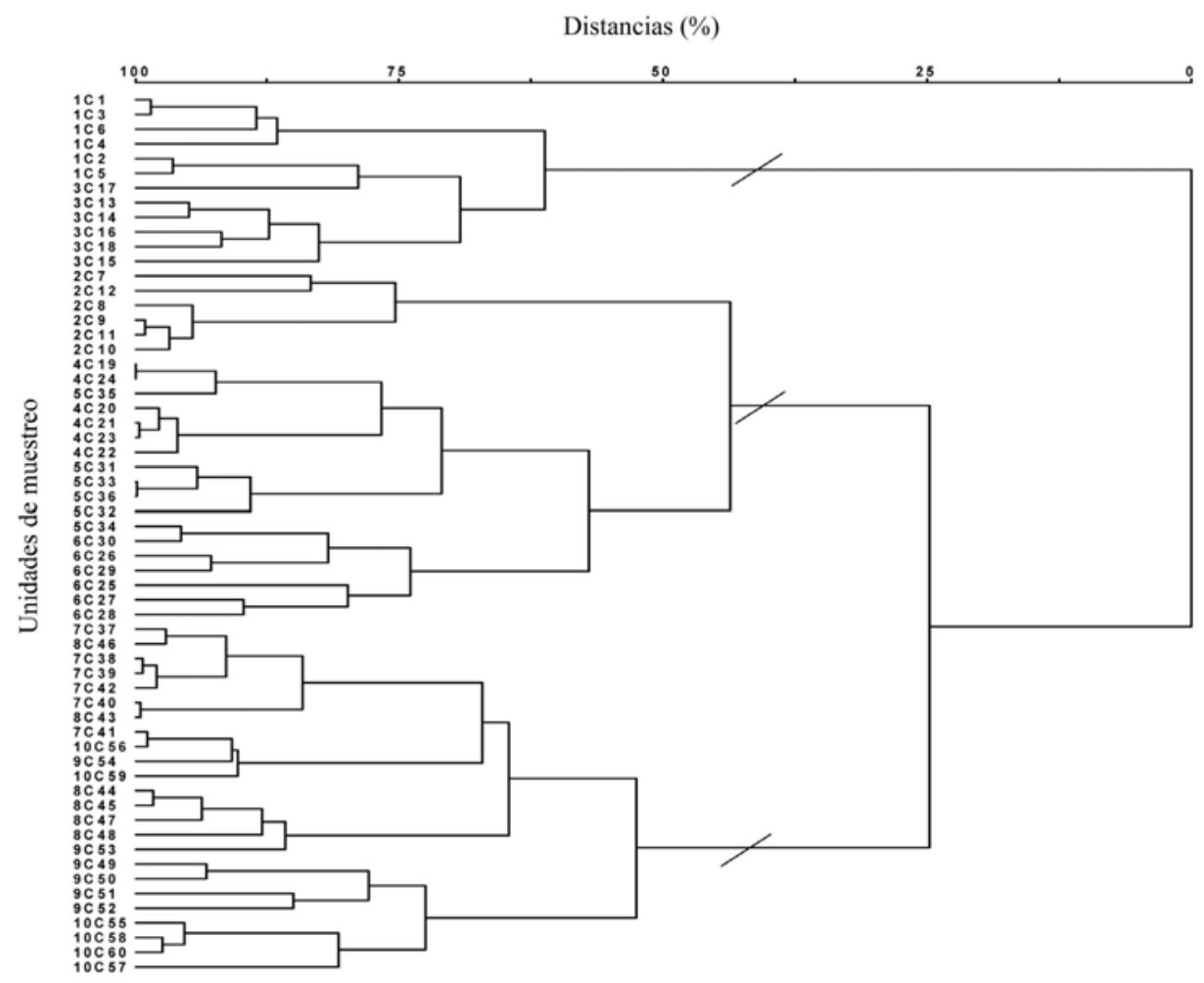

Figura 4. Diagrama de los cuadrantes de muestreo, utilizando una matriz con los valores de VIR (valor de importancia relativo) y el índice de Sørensen como medida de distancia.

nos similares fueron El Cuyo y Chuburná con un $38 \%$ de similitud, compartiendo sólo 9 especies (Cuadro 3).

Clasificación. El análisis de clasificación a un nivel de corte del $45 \%$ de la información retenida permitió distinguir tres grupos bien definidos del matorral, analizando los 60 cuadros de muestreo (Figura 4). El grupo 1 (G1) está formado por las localidades de Celestún y Chuburná, las cuales se ubican, de manera general en la parte oeste del litoral del estado; en éstas las especies que presentan un mayor valor de importancia relativa, fueron Pithecellobium keyense, Sideroxylon americanum y Capparis incana; El grupo 2 (G2) está constituido por las localidades de Sisal, Tulix, San Benito y La Casona, las cuales se ubican en la parte centro del litoral, las especies más importantes para este grupo fueron Coccothrinax readii, Bravaisia berlandieriana y Metopium brownei, con excepción de la comunidad de Sisal en la cual no se presentó $C$. readii. En el G3 están incluidas las localidades de Holchit, Punta Cancunito, Alegrías y El Cuyo, siendo las especies más importantes para este grupo Coccothrinax readii, Thrinax radiata y Pseudophoenix sargentii. El método MRPP muestra que estos tres grupos difieren significativamente en su composición florística $(P=0.0001)$.

Ordenación. En el Diagrama de Ordenación de los 60 cuadros de muestreo y los elementos del clima generado con el análisis NMDS, muestra claramente tres diferentes grupos bien definidos, los cuales corresponden a las mismas localidades que conforman los grupos obtenidos en la clasificación, en donde también se pueden observar las líneas de tendencia. La correlación entre el primer eje de ordenación y las cinco variables ambientales analizadas mostró que la precipitación presenta una alta correlación de Kendall (-0.759), seguida por la temperatura máxima (0.545), la oscilación (0.452) y la temperatura media (0.417). También se observa que el grupo G3 se ajusta con valores altos de precipitación y el grupo G1 con los valores bajos. Asimismo se observa que el grupo G1 se asocia con los valores altos de oscilación térmica y la temperatura máxima y la temperatura media; mientras que el grupo G3 se asocia con valores bajos de estas variables ambientales y el grupo G2 no presenta una asociación clara con ninguna de las cinco variables ambientales (Figura 5).

\section{Discusión}

La composición florística en relación con las formas de vida encontradas en este estudio, corrobora que la estructura de la vegetación analizada corresponde a una comunidad de matorral. Los patrones de altura obtenidos se relacionan con los que reporta Espejel (1984) presentándose el más bajo en las zonas más áridas y el matorral alto en la zona con 


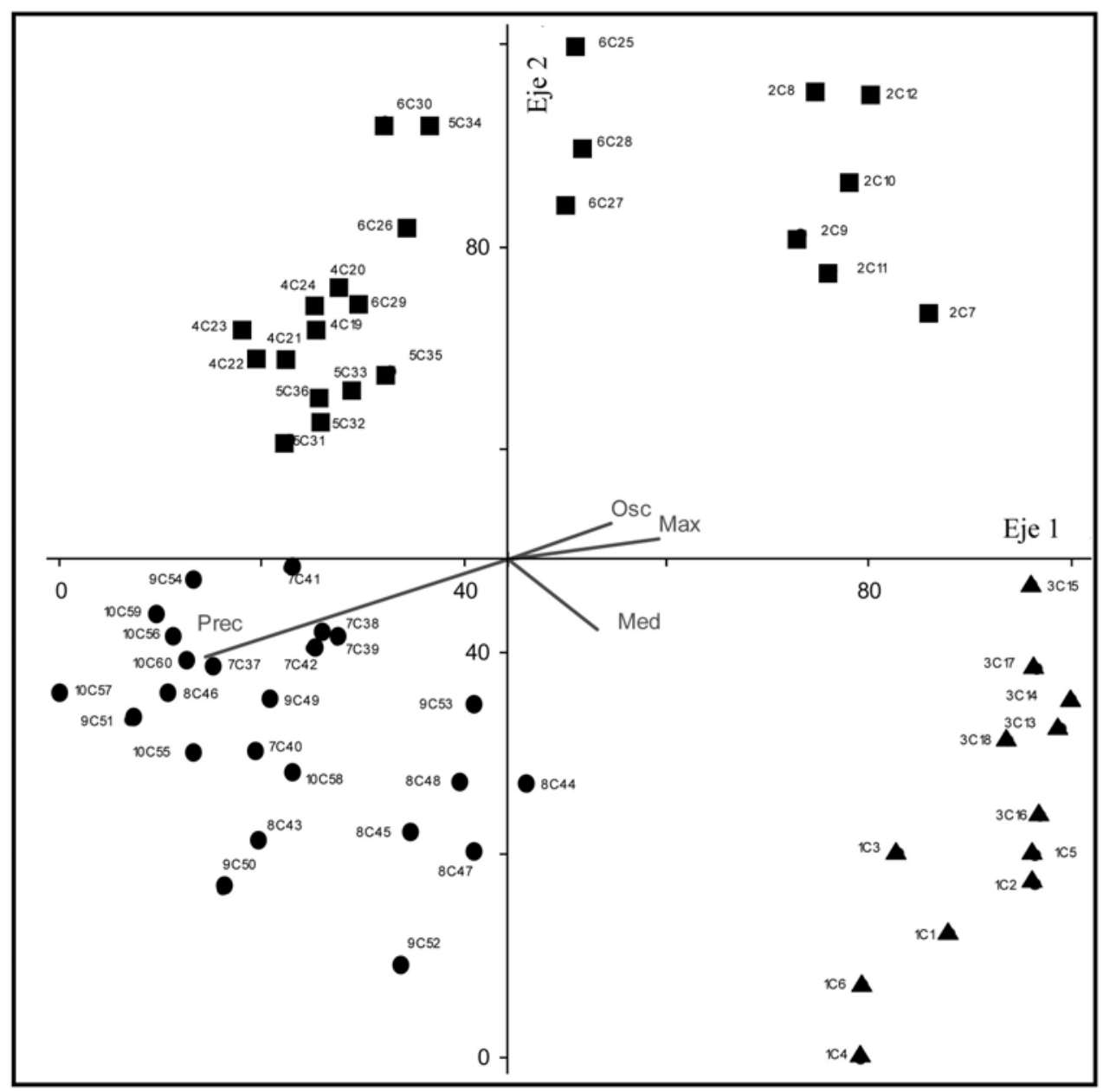

Figura 5. Diagrama de la ordenación de los 60 cuadros de muestreo producido por el análisis NMDS. Prec $=$ precipitación, Max $=$ temperatura máxima, Med = temperatura media, Min = temperatura mínima y Osc $=$ oscilación. $\mathrm{G} 1=\boldsymbol{\Delta}, \mathrm{G} 2=\mathbf{\square}$ y G3= $\mathbf{0}$.

la mayor humedad de los suelos, en la parte este y oeste del litoral. También muestra que conforme se incrementa el número de individuos de alturas mayores, disminuye el número de individuos de menor talla.

En cuanto a la riqueza de especies encontrada, ésta fue menor a la reportada por Espejel (1984) en un trabajo realizado en este mismo tipo de vegetación; ambos trabajos comparten 61 especies de 161 encontradas en el estudio anterior. Cabe señalar que no se utilizó el mismo tipo de muestreo, ya que en este estudio se emplearon cuadrantes sin incluir a los individuos menores de $50 \mathrm{~cm}$, en tanto que Espejel (1984) utilizó transectos y tomó en cuenta a las especies de pastos y herbáceas de menor talla. Adicionalmente habría que considerar los cambios nomenclaturales de algunas especies, cabe recalcar que en este trabajo se registraron 19 especies que en el estudio previo no se encontraron.

Halffter (1998) y Halffter y Moreno (2006) señalan que en el conocimiento de la diversidad alfa, beta y gamma, se debe considerar en las iniciativas de conservación de las plantas a nivel local y de paisaje, y que la persistencia de la riqueza de especies en sus hábitats y corredores biológicos en un paisaje puede contribuir a la conservación de la biodiversidad. La diversidad beta obtenida en este trabajo nos sugiere que en cada comunidad existe una buena representación florística del matorral de duna costera, pero a la vez hay un importante recambio de especies, como producto de la heterogeneidad que se presenta a lo largo del litoral.

El índice de similitud entre las localidades se obtuvo incorporando los datos de VIR de las especies; ya que la similitud entre dos comunidades puede estar basada no solamente en la composición de especies de ambas comunidades o en la abundancia que presenta cada una de las especies de éstas. Se obtiene un mayor peso en el análisis si se incluyen otros parámetros como los que considera el VIR (cobertura, densidad y frecuencia) (Mueller-Dombois y Ellermberg, 1974; Durán, 1986).

Los resultados de la clasificación nos sugieren que el matorral de duna costera que se ubica a lo largo del litoral 
yucateco presenta tres tipos de comunidades que difieren en su composición y estructura, lo cual podría estar determinado por las condiciones bióticas y abióticas en las que se encuentra cada comunidad. Espejel (1984) menciona que la distribución de algunas especies en el matorral de dunas es muy localizada y característica de ciertos lugares debido principalmente a dos factores: a) la influencia de las islas del Caribe y b) el gradiente edáfico, donde los componentes químicos del suelo, así como el agua dulce, determinan la presencia o ausencia del ciertas especies.

De acuerdo al análisis de ordenación, la composición y estructura de la vegetación del matorral de duna costera está asociada a los elementos del clima, lo que puede explicar la distribución específica de algunas especies que se limitan a una sola zona, como es el caso de Pseudophoenix sargentii en el oriente del litoral. De igual forma, nos ayuda a entender la dominancia de ciertas especies en una o varias zonas del litoral, que corresponden a los grupos de vegetación obtenidos en el análisis de clasificación. Similar a lo encontrado en este estudio, Miller et al. (2010) reportan que cada hábitat de la duna costera de la Isla de San Jorge, en Florida, está influenciado por diferentes variables del clima, en el caso de las dunas fijas la precipitación presenta una mayor influencia que la temperatura. Asimismo, Castillo et al. (1991) señalan que las diferencias encontradas en composición entre las dunas de Tabasco y Campeche podrían explicarse por factores climáticos, ya que Tabasco presenta una prolongada estación de lluvias a diferencia de Campeche que es una zona más seca. Considerando las observaciones obtenidas en estudios anteriores y en éste podemos decir que la composición de la vegetación de duna costera del litoral yucateco está fuertemente influenciada por los niveles de precipitación.

Escamilla (1999) señala que las diferencias en la composición y estructura de las comunidades vegetales, también pueden estar influenciadas por el factor suelo, debido a sus características físicas y químicas como $\mathrm{pH}$, materia orgánica, disponibilidad de nutrimentos, textura, profundidad y origen. De hecho, Espejel (1986) señala que la vegetación costera de la Península de Yucatán, en un gradiente de la playa a las dunas fijas, muestra una correlación con los factores del suelo, principalmente con el $\mathrm{pH}$, el cual disminuye al aumentar la cantidad de materia orgánica que contiene el suelo. También observó que a lo largo del litoral la capacidad de intercambio catiónico es alto en las dunas del oeste y centro del litoral, ya que en estos lugares se presenta un clima semiárido, en tanto que en las dunas del este donde el clima es más húmedo su intercambio catiónico es bajo. De igual forma, Kim y Yu (2009) reportan que los patrones de distribución de la vegetación de dunas costeras de Korea también están estrechamente relacionados con las condiciones del suelo.

Asimismo, los resultados de clasificación y ordenación muestran que hay una importante heterogeneidad entre las comunidades, tanto en la composición de especies como en la estructura de la vegetación, lo que sugiere que para la conservación de la vegetación de duna costera de Yucatán y la riqueza florística que alberga, no bastaría con conservar una sola comunidad, sino que es necesario proteger al menos una fracción de vegetación de cada una de las tres comunidades encontradas a largo del litoral yucateco (oeste, centro y este del litoral), sobretodo si se considera que en cada una de las comunidades estudiadas se encuentran algunas especies característica de cada sitio.

A pesar de que la vegetación de dunas de Yucatán se encuentra protegida por las reservas de Ría Lagartos y Ría Celestún, en sus extremos oriente y poniente del litoral yucateco, nuestro estudio muestra que las comunidades de la zona central son diferentes en composición, estructura y diversidad, siendo estas comunidades las más afectadas por el desarrollo urbano y las consecuentes actividades humanas en la zona costera (Durán et al., en proceso). Esta información ha sido de utilidad en la generación de propuestas de Unidades de Gestión Ambiental del Programa de Ordenamiento Ecológico del Territorio Costero del Estado de Yucatán (Euán-Ávila et al., 2007).

\section{Agradecimientos}

Este trabajo fue financiado por el proyecto Evaluación, Diagnóstico y Restauración de la Vegetación de la Duna Costera en el Norte de Yucatán (T-51), SEMARNAT-APIUADY. A Gabriel Dzid, quien apoyó de manera importante el trabajo de campo. A Juan Manuel Dupuy por su apoyo y sugerencias en el análisis de los datos.

\section{Literatura citada}

Campos-Rios G. y Durán R. 1991. La vegetación de la Península de Yucatán. En: Colunga P., Orellana R., Ayora N., Arellano J. y Campos G. Eds. Apuntes del Curso-Taller para maestros "El Jardín Botánico como Herramienta Didáctica”, Centro de Investigación Científica de Yucatán, Mérida, México.

Castillo S., Popma J. y Moreno-Casasola P. 1991. Coastal sand dune vegetation of Tabasco and Campeche, Mexico. Journal of vegetation science 2:73-88

Curtis J.T. y McIntosh R.P. 1951. An upland forest continuum in the prairie-forest border region of Wisconsin. Ecology 32:476496

Duch-Gary J. 1988. La Conformación Territorial de Estado de Yucatán. Universidad Autónoma Chapingo. México.

Duch-Gary J. 1991. Fisiografía del Estado de Yucatán: su Relación con la Agricultura. Universidad Autónoma Chapingo. México.

Durán R. 1986. Estudio de la vegetación de la selva baja subcaducifolia de Pseudophoenix sargentii. Tesis de Licenciatura, Facultad de Ciencias, Universidad Autónoma México, México, D.F, 77 pp.

Durán R. 1995. Diversidad florística de los petenes de Campeche. Acta Botánica Mexicana 31:73-84.

Durán R., Méndez M., Dorantes E.A. y Dzib G. 2007. Evaluación, 
diagnóstico y restauración de la vegetación y flora nativa de la duna costera en el norte de Yucatán, Informe final, SEMARNAT, Mérida, México.

Durán R., Torres W. y Espejel I. En Prensa. La vegetación de la duna costera del estado de Yucatán. En: Durán R., Méndez M. y Arellano J. Eds. Diversidad biológica y desarrollo humano en Yucatán. CICY, SEDUMA PNUD, CONABIO.

Durán R., García G. y Méndez M. En proceso. El estado de la vegetación. En: Euán-Ávila, García, A., Lizeaga-Correa, M. y Munguía A. Eds. La costa del Estado de Yucatán en el contexto de su ordenamiento ecológico. CINVESTAV, Unidad Mérida, Yucatán.

Escamilla J.A. 1999. Estudios de suelo y de ecosistemas vegetales. En: Orellana R., Escamilla J.A. y Larqué-Saavedra A. Eds. Ecofisiología Vegetal y Conservación de Recursos Genéticos, pp.119-125, Centro de Investigación Científica de Yucatán, A.C, Mérida, México.

Espejel I. 1984. La vegetación de las dunas costeras de la península de Yucatán. I. análisis florístico del estado de Yucatán. Biótica 9:183-210

Espejel I. 1986. Studies on coastal sand dune vegetation of the Yucatan Peninsula. Thesis doctoral, Uppsala University, 218 p.

Euán-Ávila J., García A. y Liceaga-Correa M.A. 2007. Programa de Ordenamiento Ecológico del Territorio Costero del Estado de Yucatán CINVESTAV, CICY, ITC, ITM, UADY. Informe técnico final. 〈www.bitacoraordenamiento.yucatan.gob.mx>

Euán A.J., Cuevas J.A. y Maldonado R.A. En Prensa. Litoral del Estado de Yucatán. En: Euán-Ávila J., García A., Liceaga-Correa M.A. y Munguía, A. Eds. La costa del estado de Yucatán en el contexto de su ordenamiento ecológico. CINVESTAV, Unidad Mérida, Yucatán.

Flores S. y Espejel I. 1994., Tipos de vegetación de la Península de Yucatán. Universidad Autónoma de Yucatán. Etnoflora Yucatanense Fasciculo 3. Yucatán, México.

Flores S., Batllori-Sampedro E., Villasuso P.M. y Mendoza-Millan M.A. 1995. Marco de referencia para el manejo de la zona costera del estado de Yucatán. Departamento Editorial de la Dirección General de Extensión de la Universidad Autónoma de Yucatán. México.

Halffter G.1998. A strategy for measuring landscape biodiversity. Biology International 36:3-17.

Halffter G. y Moreno C.E. 2006. Significado biológico de las diversidades alfa, beta y gamma. En: Halffter G., Soberón J., Koleff P. y Melic A. Eds. Sobre Diversidad Biológica: El significado de las Diversidades Alfa, Beta y Gamma, pp.5-18 m3m: Monografías Tercer Milenio vol 4, Zaragoza, España.

Hill M.O. 1973. Diversity and evenness: a unifying notation and its consequences. Ecology 54:427-432

Kim D. y Yu K.B. 2009. A conceptual model of coastal dune ecology synthesizing spatial gradients of vegetation, soil and geomorphology. Plant Ecology 202:135-148

Lortie C.J. y Cushman J.H. 2007. Effects of a directional abiotic gradient on plant community dynamics and invasion in a coastal dune system. Journal of ecology 95:468-481

Magurran A.E. 1988. Ecological Diversity and its Measurement. Princeton University Press. Cambridge.

Martínez M.L., Moreno-Casasola P. y Castillo S. 1993. Biodiversidad Costera: Playas y Dunas En: Salazar-Vallejo S. I. y González N.E. Eds. Biodiversidad Marina y Costera de México, pp.160-181 CONABIO y CIQRO, México.

McCune B. y Mefford M. J. 1999. PC-ORD. Multivariate Analysis of Ecological Data V.4.0. MjM Software Design, Gleneden Beach, Oregon.

Miller T.E., Gornish E.S. y Buckley H.L. 2010. Climate and coastal dune vegetation: disturbance, recovery and succession. Plant ecology 206:97-104.

Miranda F. 1978. Vegetación de la Península Yucateca. Colegio de Postgraduados. Chapingo México. $271 \mathrm{p}$.

Moreno-Casasola P., Espejel I., Castillo S., Castillo-Campos G., Durán R., Pérez-Navarro J.J., León J.L., Olmsted I. y TrejoTorres J. 1998. Flora de los ambientes arenosos y rocosos de las costas de México. En: Halffter G. (Comp.). La Diversidad Biológica de Iberoamérica Vol. II, pp.177-258 Acta Zoológica Mexicana, nueva serie, Instituto de Ecología, A.C., Xalapa, México.

Mueller-Dombois D. y Ellenberg H. 1974. Aims and Methods of Vegetation Ecology. Wiley Internacional. United States of America.

Orellana R., Balam K.M., Bañuelos I., García M.E., GonzálezIturbe J.A., Herrera C.F. y Vidal L.J. 1999. Evaluación climática. En: García de Fuentes A. y Córdoba-Ordoñez J. Eds. Atlas de Procesos Territoriales de Yucatán, pp.163-182 Universidad Autónoma de Yucatán, México.

Primack R., Roíz R., Feinsinger P., Dirzo R. y Masssoardo F. 2001. Fundamentos de Conservación Biológica. Perspectivas Latinoamericanas. Fondo de Cultura Económica. D.F. México.

Rzedowski J. 1978. Vegetación de México. Limusa. DF, México.

Servicio Meteorológico Nacional, CONAGUA. 2007. Precipitación y Temperatura de Yucatán, en: 〈www.sma.cna.gob.mx〉.

Sørensen T. 1948. A method of establishing groups of equal amplitude in plant sociology based on similarity of species content. Biologiske Skrifter 5:1-34

Torres L.N. y Bojórquez L.H. 2002. Las dunas costeras de Veracruz: Un paisaje amenazado. Revista Higiene 4:7-15.

Whittaker R.H. 1972. Evolution and measurement of species diversity. Taxon 21:213-251.

Wolfe S.A. y Nickling W.G. 1993. The protective role of sparse vegetation in wind erosion. Progress in Physical Geography 17:50-68. 
Apéndice 1. Valor de Importancia relativa (VIR) de las primeras 10 especies cada una de las localidades.

\begin{tabular}{|c|c|c|c|c|}
\hline Especie & $\begin{array}{c}\text { Cobertura relativa } \\
\%\end{array}$ & $\begin{array}{c}\text { Densidad relativa } \\
\% \%\end{array}$ & $\begin{array}{c}\text { Frecuencia relativa } \\
\%\end{array}$ & V.I.R \\
\hline \multicolumn{5}{|l|}{ Celestún } \\
\hline Pithecellobium keyense & 29.63 & 21.88 & 7.69 & 59.20 \\
\hline Enriquebeltrania crenatifolia & 18.19 & 11.11 & 5.13 & 34.43 \\
\hline Sideroxylon americanum & 22.69 & 4.17 & 6.41 & 33.26 \\
\hline Capparis incana & 11.16 & 6.94 & 6.41 & 24.51 \\
\hline Rivina humilis & 1.78 & 9.72 & 7.69 & 19.20 \\
\hline Malvaviscus arboreus & 1.47 & 7.64 & 7.69 & 16.80 \\
\hline Thrinax radiata & 3.25 & 7.29 & 5.13 & 15.67 \\
\hline Gomphrena serrata & 2.97 & 5.56 & 6.41 & 14.94 \\
\hline Jacquinia macrocarpa & 1.18 & 5.56 & 6.41 & 13.14 \\
\hline Chiococca alba & 1.18 & 4.86 & 6.41 & 12.45 \\
\hline \multicolumn{5}{|l|}{ Sisal } \\
\hline Bravaisia berlandieriana & 38.43 & 23.23 & 8.70 & 70.35 \\
\hline Metopium brownei & 21.57 & 17.42 & 8.70 & 47.68 \\
\hline Pithecellobium keyense & 5.91 & 9.03 & 8.70 & 23.63 \\
\hline Sideroxylon americanum & 15.59 & 2.58 & 4.35 & 22.52 \\
\hline Gomphrena serrata & 5.18 & 3.87 & 7.25 & 16.30 \\
\hline Jacquinia macrocarpa & 1.02 & 5.81 & 7.25 & 14.07 \\
\hline Rivina humilis & 0.41 & 4.52 & 5.80 & 10.72 \\
\hline Capparis flexuosa & 0.28 & 3.23 & 5.80 & 9.30 \\
\hline Hymenocallis littoralis & 1.32 & 4.52 & 2.90 & 8.74 \\
\hline Malvaviscus arboreus & 0.59 & 3.23 & 4.35 & 8.16 \\
\hline \multicolumn{5}{|l|}{ Chuburná } \\
\hline Pithecellobium keyense & 44.10 & 13.40 & 7.79 & 65.29 \\
\hline Sideroxylon americanum & 18.41 & 8.76 & 7.79 & 34.96 \\
\hline Acanthocereus tetragonus & 11.45 & 8.25 & 7.79 & 27.49 \\
\hline Capparis incana & 7.91 & 10.82 & 7.79 & 26.53 \\
\hline Bravaisia berlandieriana & 3.03 & 12.37 & 6.49 & 21.89 \\
\hline Justicia carthaginensis & 1.06 & 11.34 & 3.90 & 16.30 \\
\hline Crossopetalum rhacoma & 0.68 & 5.15 & 6.49 & 12.33 \\
\hline Rivina humilis & 0.71 & 3.09 & 6.49 & 10.29 \\
\hline Jacquinia macrocarpa & 0.79 & 2.58 & 5.19 & 8.56 \\
\hline Capparis flexuosa & 0.81 & 2.58 & 3.90 & 7.28 \\
\hline \multicolumn{5}{|l|}{ Tulix } \\
\hline Coccothrinax readii & 43.67 & 36.53 & 6.59 & 86.80 \\
\hline Bravaisia berlandieriana & 16.92 & 22.65 & 6.59 & 46.17 \\
\hline Metopium brownei & 9.62 & 4.69 & 6.59 & 20.91 \\
\hline Gymnanthes lucida & 5.48 & 7.55 & 6.59 & 19.63 \\
\hline Sideroxylon americanum & 5.01 & 3.06 & 5.49 & 13.57 \\
\hline Diospyros cuneata & 1.89 & 3.88 & 6.59 & 12.36 \\
\hline Anthurium schlechtendalii & 2.91 & 2.86 & 5.49 & 11.26 \\
\hline Capparis incana & 0.81 & 1.84 & 6.59 & 9.24 \\
\hline Crossopetalum rhacoma & 1.37 & 2.86 & 4.40 & 8.62 \\
\hline Enriquebeltrania crenatifolia & 2.29 & 1.22 & 4.40 & 7.91 \\
\hline
\end{tabular}


Wendy Torres, Martha Méndez, Alfredo Dorantes y Rafael Durán

\begin{tabular}{|c|c|c|c|c|}
\hline Especie & $\begin{array}{c}\text { Cobertura relativa } \\
\%\end{array}$ & $\begin{array}{c}\text { Densidad relativa } \\
\%\end{array}$ & $\begin{array}{l}\text { Frecuencia relativa } \\
\qquad \%\end{array}$ & V.I.R \\
\hline \multicolumn{5}{|l|}{ San Benito } \\
\hline Coccothrinax readii & 24.81 & 28.02 & 6.32 & 59.15 \\
\hline Bravaisia berlandieriana & 15.00 & 19.82 & 6.32 & 41.13 \\
\hline Metopium brownei & 14.89 & 10.71 & 6.32 & 31.91 \\
\hline Thrinax radiata & 18.42 & 5.92 & 6.32 & 30.65 \\
\hline Porophyllum punctatum & 2.85 & 4.78 & 6.32 & 13.95 \\
\hline Hippocratea excelsa & 4.97 & 3.87 & 4.21 & 13.05 \\
\hline Coccoloba uvifera & 5.91 & 2.73 & 4.21 & 12.86 \\
\hline Malvaviscus arboreus & 0.95 & 2.51 & 4.21 & 7.66 \\
\hline Crossopetalum rhacoma & 0.69 & 1.59 & 5.26 & 7.55 \\
\hline Diospyros cuneata & 0.82 & 1.82 & 4.21 & 6.85 \\
\hline \multicolumn{5}{|l|}{ La Casona } \\
\hline Metopium brownei & 21.77 & 15.31 & 5.83 & 42.90 \\
\hline Coccothrinax readii & 17.40 & 19.39 & 5.83 & 42.61 \\
\hline Bravaisia berlandieriana & 7.34 & 11.22 & 5.83 & 24.39 \\
\hline Caesalpinia vesicaria & 14.52 & 3.57 & 4.85 & 22.95 \\
\hline Coccoloba uvifera & 12.77 & 4.34 & 5.83 & 22.94 \\
\hline Porophyllum punctatum & 2.54 & 6.12 & 5.83 & 14.49 \\
\hline Malvaviscus arboreus & 2.38 & 5.87 & 4.85 & 13.10 \\
\hline Justicia carthaginensis & 1.53 & 5.61 & 3.88 & 11.02 \\
\hline Crossopetalum rhacoma & 1.67 & 4.08 & 4.85 & 10.60 \\
\hline Capparis incana & 1.10 & 2.55 & 5.83 & 9.48 \\
\hline \multicolumn{5}{|l|}{ Holchit } \\
\hline Coccothrinax readii & 27.73 & 34.48 & 8.22 & 70.43 \\
\hline Thrinax radiata & 20.15 & 20.69 & 8.22 & 49.05 \\
\hline Pseudophoenix sargentii & 14.71 & 10.00 & 8.22 & 32.93 \\
\hline Metopium brownei & 10.00 & 3.10 & 6.85 & 19.95 \\
\hline Enriquebeltrania crenatifolia & 7.07 & 3.79 & 6.85 & 17.71 \\
\hline Sideroxylon americanum & 8.84 & 2.76 & 5.48 & 17.08 \\
\hline Pithecellobium keyense & 3.83 & 3.45 & 6.85 & 14.13 \\
\hline Capparis incana & 0.72 & 3.45 & 6.85 & 11.02 \\
\hline Jacquinia macrocarpa & 0.41 & 2.76 & 6.85 & 10.02 \\
\hline Tillandsia dasyliriifolia & 1.25 & 2.76 & 5.48 & 9.49 \\
\hline \multicolumn{5}{|l|}{ Punta Cancunito } \\
\hline Coccothrinax readii & 24.40 & 31.27 & 8.00 & 63.67 \\
\hline Thrinax radiata & 18.30 & 21.09 & 8.00 & 47.39 \\
\hline Pithecellobium keyense & 23.90 & 11.27 & 8.00 & 43.17 \\
\hline Pseudophoenix sargentii & 10.79 & 7.64 & 8.00 & 26.43 \\
\hline Acanthocereus tetragonus & 3.09 & 4.00 & 8.00 & 15.09 \\
\hline Vallesia antillana & 3.51 & 2.55 & 6.67 & 12.72 \\
\hline Enriquebeltrania crenatifolia & 3.29 & 2.55 & 5.33 & 11.16 \\
\hline Tillandsia dasyliriifolia & 1.19 & 3.27 & 5.33 & 9.80 \\
\hline Agave angustifolia & 1.30 & 2.55 & 5.33 & 9.17 \\
\hline Jacquinia macrocarpa & 0.67 & 1.82 & 6.67 & 9.16 \\
\hline
\end{tabular}


DivERSIDAD DEL MATORRAL COSTERO EN EL LITORAL YUCATECO

\begin{tabular}{|c|c|c|c|c|}
\hline Especie & $\begin{array}{c}\text { Cobertura relativa } \\
\%\end{array}$ & $\begin{array}{c}\text { Densidad relativa } \\
\%\end{array}$ & $\begin{array}{c}\text { Frecuencia relativa } \\
\% \%\end{array}$ & V.I.R \\
\hline \multicolumn{5}{|l|}{ Alegrías } \\
\hline Coccothrinax readii & 19.21 & 15.43 & 6.19 & 40.83 \\
\hline Thrinax radiata & 21.12 & 12.35 & 5.15 & 38.62 \\
\hline Pseudophoenix sargentii & 16.13 & 12.96 & 6.19 & 35.28 \\
\hline Bravaisia berlandieriana & 6.81 & 11.11 & 6.19 & 24.10 \\
\hline Gymnanthes lucida & 7.06 & 9.88 & 5.15 & 22.09 \\
\hline Manilkara zapota & 11.25 & 2.16 & 5.15 & 18.56 \\
\hline Agave angustifolia & 3.64 & 5.56 & 4.12 & 13.32 \\
\hline Neea psychotrioides & 2.34 & 4.63 & 6.19 & 13.15 \\
\hline Diospyros cuneata & 0.62 & 3.70 & 5.15 & 9.48 \\
\hline Malvaviscus arboreus & 1.30 & 4.01 & 4.12 & 9.43 \\
\hline \multicolumn{5}{|l|}{ El Cuyo } \\
\hline Coccothrinax readii & 19.76 & 17.31 & 5.77 & 42.84 \\
\hline Pseudophoenix sargentii & 17.57 & 12.84 & 5.77 & 36.18 \\
\hline Gymnanthes lucida & 11.91 & 11.34 & 5.77 & 29.02 \\
\hline Thrinax radiata & 13.07 & 10.15 & 4.81 & 28.03 \\
\hline Bravaisia berlandieriana & 4.70 & 9.25 & 5.77 & 19.73 \\
\hline Pithecellobium keyense & 4.86 & 3.88 & 5.77 & 14.50 \\
\hline Neea psychotrioides & 2.75 & 4.18 & 5.77 & 12.70 \\
\hline Eugenia axillaris & 2.71 & 3.58 & 4.81 & 11.10 \\
\hline Diospyros cuneata & 2.88 & 3.28 & 3.85 & 10.01 \\
\hline Jacquinia macrocarpa & 1.59 & 3.58 & 4.81 & 9.98 \\
\hline
\end{tabular}


Apéndice 2. Lista de especies

Acanthaceae

Agavaceae

Amaranthaceae

Amaryllidaceae

Anacardiaceae

Asclepiadaceae

Apocynaceae

Araceae

Arecaceae

Asteraceae

Boraginaceae

Capparaceae

Bromeliaceae

Burseraceae

Cactaceae

Celastraceae

Commelinaceae

Ebenaceae
Bravaisia berlandieriana (Nees) T.F. Daniel

Dicliptera sexangularis (L.) Juss.

Justicia carthaginensis Jacq.

Agave angustifolia Haw.

Gomphrena serrata L.

Iresine celosia L.

Hymenocallis littoralis Salisb.

Metopium brownei Urb.

Cynanchum schlechtendalii (Decne.) Standl. \& Steyerm.

Macroscepis diademata (Ker Gawl.) W.D. Stevens

Echites umbellatus Jacq.

Thevetia gaumeri Hemsl.

Vallesia antillana Woodson

Anthurium schlechtendalii Kunth

Coccothrinax readii H.J. Quero

Pseudophoenix sargentii $\mathrm{H}$. Wendl.

Thrinax radiata Lodd. Ex Desf.

Baccharis dioica Vahl

Bidens alba DC.

Chromolaena lundellii R.M. King \& H.Rob.

Flaveria linearis Lag.

Melanthera aspera (Jacq.) Small

Porophyllum punctatum S.F. Blake

Cordia globosa Kunth

Capparis flexuosa (L.) L.

Capparis incana Kunth

Capparis indica (L.) Druce

Tillandsia dasyliriifolia Baker

Bursera simaruba (L.) Sarg.

Acanthocereus tetragonus (L.) Hummelinck

Nopalea gaumeri Britton \& Rose

Opuntia dillenii Haw.

Selenicereus donkelaarii Britton \& Rose

Crossopetalum rhacoma Hitchc.

Hippocratea excelsa Kunth

Maytenus phyllantoides Benth.

Commelina erecta L.

Diospyros cuneata Standl. 


\begin{tabular}{|c|c|}
\hline Euphorbiaceae & $\begin{array}{l}\text { Enriquebeltrania crenatifolia (Miranda) Rzed. } \\
\text { Euphorbia heterophylla L. } \\
\text { Gymnanthes lucida Sw. }\end{array}$ \\
\hline Fabaceae & $\begin{array}{l}\text { Caesalpinia vesicaria L. } \\
\text { Piscidia piscipula (L.) Sarg. } \\
\text { Pithecellobium keyense Britton ex Coker }\end{array}$ \\
\hline Malpighiaceae & Malpighia souzae Miranda \\
\hline Malvaceae & $\begin{array}{l}\text { Gossypium hirsutum L. } \\
\text { Malvaviscus arboreus Cav. } \\
\text { Waltheria americana L. }\end{array}$ \\
\hline Menispermaceae & Hyperbaena winzerlingii Standl. \\
\hline Myrtaceae & Eugenia axillaris Willd. \\
\hline Nyctaginaceae & $\begin{array}{l}\text { Boerharvia diffusa L. } \\
\text { Commicarpus scandens (L.) Standl. } \\
\text { Neea psychotrioides Donn. Sm. } \\
\text { Neea choriophylla var hirtella Lundell }\end{array}$ \\
\hline Orchidaceae & $\begin{array}{l}\text { Catasetum integerrimum Hook } \\
\text { Cyrtopodium macrobulbum (La Llave \& Lex.) G.A. Romero \& Carnevali }\end{array}$ \\
\hline Passifloraceae & $\begin{array}{l}\text { Passiflora foetida L. } \\
\text { Passiflora suberosa L. }\end{array}$ \\
\hline Phytolaccaceae & Rivina humilis L. \\
\hline Poaceae & Lasiacis divaricata (L.) Hitchc. \\
\hline Polygonaceae & Coccoloba uvifera L. \\
\hline Rhamnaceae & Krugiodendron ferreum Urb. \\
\hline Rubiaceae & $\begin{array}{l}\text { Chiococca alba Hitchc. } \\
\text { Chiococca coriacea M. Martens \& Galeotti } \\
\text { Ernodea littoralis Sw. }\end{array}$ \\
\hline Sapotaceae & $\begin{array}{l}\text { Sideroxylon americanum (Mill.) T.D. Penn. } \\
\text { Manilkara zapota (L.) P. Royen }\end{array}$ \\
\hline Solanaceae & Lycium carolinianum Walter \\
\hline Surianaceae & Suriana maritima L. \\
\hline Theophrastaceae & Jacquinia macrocarpa Cav. \\
\hline Verbenaceae & Lantana involucrata L. \\
\hline
\end{tabular}

